\title{
Partial discharge detection device using ultrasonic sensor on medium voltage XLPE cable
}

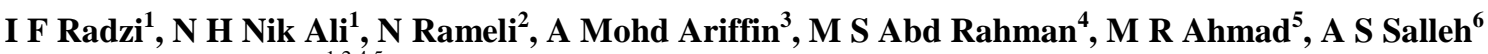 \\ ${ }_{1,2,4,5}$ College of Engineering, Universiti Tenaga Nasional, Malaysia \\ ${ }^{3}$ TNB Research, Malaysia \\ ${ }^{5}$ Department of Electrical \& Electronics Engineering, Universiti Teknologi PETRONAS, Malaysia \\ ${ }^{6}$ Universiti Teknologi Malaysia, Faculty of Computer Science, Malaysia
}

\begin{tabular}{l}
\hline \hline Article Info \\
\hline Article history: \\
Received May 22, 2019 \\
Revised Jul 24, 2019 \\
Accepted Aug 8, 2019
\end{tabular}

Keywords:

Partial discharge

Power cable

Ultrasonic Sensor

\begin{abstract}
There are many methods that have been studied by earlier researchers in order to detect the acoustic properties of Partial Discharge (PD) emitted by PD sources. One of the methods known as ultrasonic sensing on medium voltage Cross-Linked Polyethylene (XLPE) cable was adopted to detect partial discharges on commercial applications, usually by using Acoustic Emission (AE) sensors. This paper presents the processes of designing a PD sensor to detect the acoustic properties of the partial discharges on medium voltage XLPE cable. This PD sensor method works by detecting the partial discharges occurred at the joint of the cable which can act as an early warning device to help minimize the repair and maintenance costs of degrading cable. Result of the experiment shows the complete design of the prototype device, the device after fabrication and the functionality of the device. This design of the prototype can be beneficial for future uses in designing cost efficient and smaller sized PD detection devices. By positioning the sensor in horizontal position directly to the source of PD on the cable, the sensor will be able to detect acoustic properties of PD, by picking up the frequencies beyond $40 \mathrm{kHz}$. By varying the voltage applied values, a design of experiment (DOE) is carried out accordingly. Result of the experiment shows that the prototype device is functioning as expected, and hence this finding will be very useful to the consumers of power industries as the sensor device can serve as an alternative device to the commercialized PD sensing devices which are bulky and expensive.
\end{abstract}

Copyright $\odot 2020$ Institute of Advanced Engineering and Science. All rights reserved.

\section{Corresponding Author:}

Mohamad Izmir Farhan Radzi,

College of Engineering,

Universiti Tenaga Nasional,

Jalan IKRAM-UNITEN, 43000 Kajang, Selangor, Malaysia.

Email: mohamad.izmir.farhan@gmail.com

\section{INTRODUCTION}

Power cable makes up the majority of important assets in the field of electrical power system. Mostly used for transmission of electrical power, power cables are also used under variety of electrical, mechanical and environmental conditions [1]. Partial discharge (PD) analysis is important to assess and evaluate the insulation state of high voltage equipment under service in order to determine the severity of degradation [2-5]. PD is a phenomenon where a breakdown process of a small area of an insulation, which is subjected to high voltage occurs. PD is an electrical discharge that do not completely or partially bridge the insulation between conductors and which can or cannot occur adjacent to a conductor [6, 7]. It can be due to faulty design, material defects and aging assets. These factors will eventually lead to the production of 
various PD sources [8, 9]. Void discharge is a type of internal PD and it normally happens in the medium voltage cables with defects in the joints[10,11].

Nevertheless, numerous studies have confirmed that PD drastically promotes long term degradation which eventually leads to failure of electrical insulation. Majority of electrical equipment produce a broad range of sound and the most basic electrical problems produce distinct ultrasound waves which can be detected through ultrasonic testing. Furthermore, there are many kinds of sensors and methods that have been developed to assess the ultrasonic testing [12-16]. Ultrasonic measurement is one of the most powerful on a comparative basis and will significantly increase the reliability of correct detection of PD when used with other online PD testing technologies. On the other hand, with the advancement of technology especially in the development of Integrated Circuits (IC), the term operational amplifiers or op amp comes into the limelight. The concept of op amp is used as a basis in this research to design the custom Partial Discharge Detection (PDD) device. Hence, This paper presents the design process of the custom PDD device, and the functionality of the device to detect PD on medium voltage XLPE cable have been studied and presented.

\section{METHODOLOGY}

In this study, the customized PDD device is developed to detect the PD source, which comprises an ultrasonic sensor as a sensing element to detect the acoustic sound waves of a range of $40 \mathrm{kHz}$ and above. Thus, it is of utmost importance to adhere to the meticulous design process. The first design process is to create a receiver circuit, where the receiver itself is of an ultrasonic device. This receiver circuit should function in a three-step process. The first functional process is to detect the ultrasonic signal from the PD source and goes through the differential amplifier, in order for the receiver to differentiate and amplify the collected signal that varies with a gain. The second functional process is for the amplified signal to pass through a high pass filter in order to remove lower unwanted frequencies or noises. The final functional process is for the filtered signal to be analyzed by an oscilloscope. Speaking of operational amplifiers, an op amp is a device containing a circuit that consists of transistors, resistors, capacitors and diodes. It typically requires connection of bipolar power supplies which are both $+V s$ and $-V s$ with respect to ground [17]. This custom PDD device focuses on using differential amplifiers and high pass filter. An ideal differential amplifier provides an output voltage with respect to ground, that is some gain times the difference between two input voltages. The formula is expressed as following:

$$
V_{\text {out }}=A\left(V_{a}-V_{b}\right)
$$

$\mathrm{A}$ is the differential gain and both $\mathrm{V}_{\mathrm{a}}$ and $\mathrm{V}_{\mathrm{b}}$ are voltages with respect to ground. LT Spice was used to construct the circuit design of the differential amplifier, and it is as shown in Figure 1.



Figure 1. Circuit diagram of a differential amplifier designed using LT Spice, which is used in the prototype device.

The differential amplifier consists of two $10 \mathrm{k} \Omega$ resistors, two $330 \Omega$ resistors, one $5 \mathrm{k} \Omega$ variable resistor with adjustable gain, a $100 \mathrm{pF}$ capacitor, a buzzer and a 741 operational amplifier. The whole circuit design functions as a differential amplifier. The common formula for the differential amplifier in order to get the $\mathrm{V}_{\text {out }}$ is as the following:

Partial discharge detection device using ultrasonic sensor on medium voltage XLPE cable (I F Radzi) 


$$
V_{\text {out }}=\left(\frac{R_{2}}{R_{1}}\right)\left(V_{2}-V_{1}\right)
$$

The second functional process consists of a high pass filter. A high pass filter is a filter that only allows high frequency signals to pass through, and in the same time, removing the lower frequency signals. This helps to classify the collected PD signal better since it only allows a frequency of $40 \mathrm{kHz}$ and above to pass through. The circuit design of the high pass filter used in this project is shown in Figure 2.



Figure 2. Circuit diagram of a high pass filter designed using LT Spice, which is used in the prototype device

The circuit diagram consists of a $1 \mathrm{k} \Omega$ resistor, two $10 \mathrm{k} \Omega$ resistors, a 741 operational amplifier, and a $100 \mathrm{pF}$ capacitor. The $\mathrm{V}_{\text {out }}$ is the filtered PD signal, and the next step is the analysis process done using an oscilloscope. Combining the two circuits together, a functional PD detection device circuit will be obtained. Figure 3 shows the combined circuits with additional components such as voltage sources:

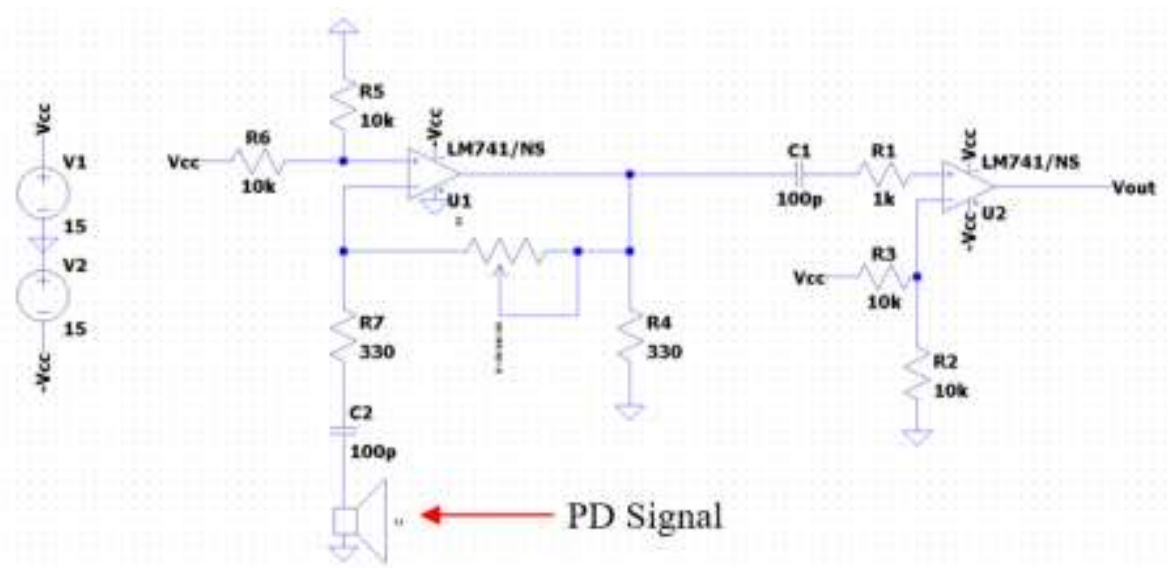

Figure 3. Combined circuit diagram of differential amplifier and high pass filter designed using LT Spice, which is used in the prototype device. External $5 \mathrm{~V}$ voltage sources to power up the device is abbreviated as $\mathrm{V}_{\mathrm{cc}}$.

With a completed design using LT Spice of the prototype device, the design can now be transferred to Autodesk EAGLE in order to construct the design of the Printed Circuit Board (PCB). In Autodesk EAGLE, a schematic diagram must be created first. Only then can the schematic diagram be converted into a PCB diagram. The LT Spice schematic diagram can be converted into Autodesk EAGLE schematic diagram format.

With the completed schematic diagram, now the design can be converted directly into a PCB diagram. The converted design is not in an orderly manner, therefore, modifications need to be done in order to get the final design. Ratsnest was clicked in order to get the shortest possible connections between the elements automatically. The Ratsnest action tab redefined the wires' connections and it created the shortest route, for the best possible design. Only one layer is used for the PCB design. Next step is to define the 
boundary of the PCB design in order to make sure the components fits in nicely onto the PCB, according to the components respective positions. Finally, the finished product of the custom PDD sensor will be fabricated based on the final design of the PCB diagram. Note that the design can still be modified if further improvements of the prototype device is to be amended. The device was fabricated and components were soldered at a fabrication company.

Figure 4 shows the fabricated prototype device which is ready to be tested for its functionality.

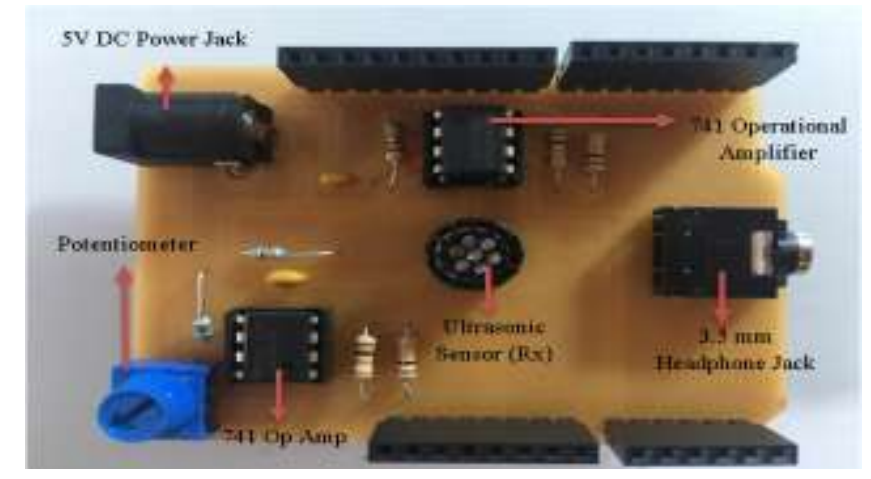

Figure 4. The fabricated prototype device known as PDD device that is ready to be tested for its functionality

\section{EXPERIMENTAL MEASUREMENT}

This section explains the details of the artificial PD source and also the setup of the experiment used in investigating the acoustic properties of PD signal measured from HFCT and the ultrasonic sensor or custom PDD sensor accordingly. The measured PD signals by the HFCT connected to the earth braid of cable joint are used for further analysis to compare the performance of the sensor. An experimental setup has been designed and constructed in the TNB Research Sdn. Bhd Laboratory to simulate void discharge inside a cable. Void discharge that has $1.15 \mathrm{~mm}$ depth and $2.93 \mathrm{~mm}$ width was generated artificially in the laboratory which then was used to locate near to insulation screen of XLPE cable as input data. Void discharge is a common type of discharge activity that occurs within a power line cable. Void discharges are common due to defects known as cavities that appears inside an electrical insulation [18]. The defects inside a cable tend to appear due to poor manufacturing errors or improper transportation of the cable. When subsequent amount of voltage equaling to the minimum inception voltage is applied, void discharges start to occur within the power cable and PD signals travel within the cable from the source location via the HFCT to the ground. A Teledyne Lecroy digital oscilloscope with a sampling rate set to $2.5 \mathrm{GSs}-1$ and a bandwidth of $500 \mathrm{MHz}$ respectively is then used for real time waveform analysis and data recording. The Channel 1 of the oscilloscope is connected to the HFCT sensor unit while the ultrasonic sensor was connected to Channel 2. The trigger on the oscilloscope was set in phase to the applied voltage. The data obtained from the HFCT sensor was then used for comparison purposes with the data obtained from the ultrasonic sensor.

\section{PARTIAL DISCHARGE EXTRACTION}

In this section, a converter was used to convert the stored data into Matlab files format (.mat) for further analyses and this is known as the initial pre-processing stage. To allow for further processing, the DC offset needs to be removed from the raw data. This is because a DC offset was produced in the measured signals due to the operation of the pulse generation hardware. This was done by subtracting the mean amplitude of the entire cycle from one of each individual sample in order to ensure that it exhibits a zero mean. For background noises, it is of utmost importance to remove the noise in order to extract PD pulses. This is because background noises affects the measurement of the PD pulses. The experimentations were carried out in a controlled laboratory condition which have a low noise level, and in order to de-noise the raw signal and extract the PD pulses, a method known as hard-thresholding [19] was used. The process of extraction was done using a well-known algorithm known as a peak detection technique. The technique is implemented onto the raw data, transferring each pulse into a new variable matrix, replacing the removed pulse with a zero vector [20,21]. To ensure the peak magnitude of the remaining pulses within the raw data is smaller than a pre-defined threshold value, this process was repeated several times. With this, 
the significant PD pulses can be isolated from background noise. Identifying the value of background noise via the signal plot of raw data which clearly show the noise level is very important before carefully setting a threshold value. If the threshold value was set too high, possibility of loss of PD pulse information will be immense. On the other hand, if the threshold value was set too low, PD events might be interpreted from noise pulses $[22,23]$. For some cases, according to particular conditions, the threshold value can be differed. Figure 5 proves that the value of the threshold was set to $1.5 \mathrm{mV}$ for both HFCT and ultrasonic sensor.
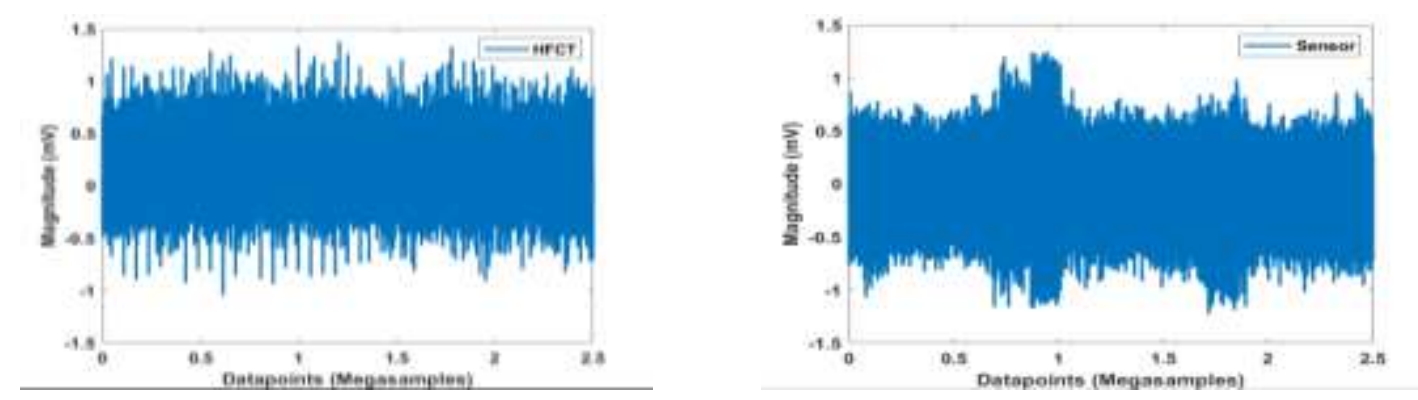

Figure 5. Threshold value was set to $1.5 \mathrm{mV}$ for both HFCT and ultrasonic sensor

The background noise and the DC offset was then removed, and the pure signal from the HFCT and PDD devices is assumed to only contain PD pulses of interest [22]. The task of noise suppression within the PD data obtained can be carried out with various de-noising techniques. The techniques are known as Fast Fourier Transform (FFT) based de-noising, wavelet de-noising and low pass filtering [24-27].

\section{RESULTS AND ANALYSIS}

In this section, before a comparison can be made, the process of finding and matching the pairs of PD pulses from the HFCT and ultrasonic sensor was carried out by using the pulse pairing process which is based on a reasonable time of flight duration. In investigating the performance of the ultrasonic sensor, a main analysis was done by comparing in time domain of measured PD pulses from both measurement techniques. Figure 6 shows the correlation of individually measured PD pulses from the two measurement techniques under an applied voltage of $6.5 \mathrm{kV}, 7.5 \mathrm{kV}$ and $8.5 \mathrm{kV}$ respectively.

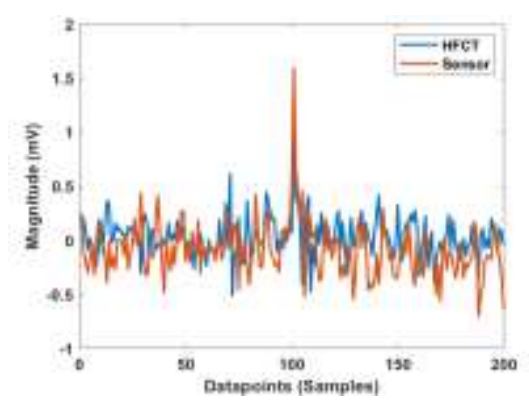

(a) $6.5 \mathrm{kV}$

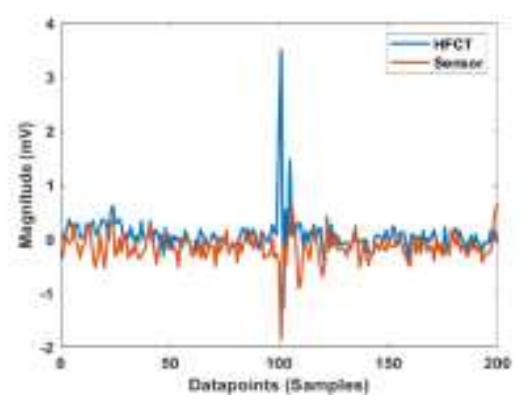

(b) $7.5 \mathrm{kV}$

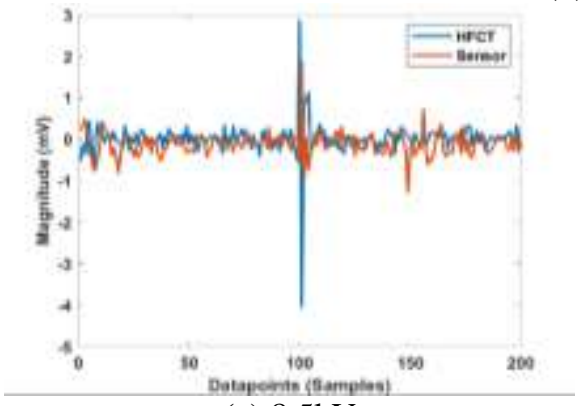

(c) $8.5 \mathrm{kV}$ 
Figure 6. Individual comparison of measured PD pulses from both measurement techniques under different values of applied voltage

Figure 6 shows that the sensor can detect most of the PD pulses detected by HFCT. However, the magnitude of measured PD signals from the HFCT is sententiously greater compared to the magnitude of pulses measured from the ultrasonic sensor. This is due to the nature of acoustic emission sensing method, which is affected by signal attenuation and extraneous noise while HFCT is not affected. Therefore, the waveform of the PD detected by ultrasonic sensor is affected, hence, the difference in magnitude. The values of PD captured are depending on the voltage applied during the experiment. Table 1 shows the maximum average magnitude of PD pulses captured by the sensor for each voltage applied. It is found that the higher the voltage injection into the cable, the higher the maximum average magnitude of the PD pulses detected by the sensor.

Even though the sensor is not able to capture the PD pulses as accurate as the HFCT sensor, ultimately the custom PDD sensor is still able to achieve the main objective of this research project, and that is being able to detect PD pulses in Medium Voltage XLPE cable due to void discharges. Further improvements of the ultrasonic sensor device will be amended in the near future if applicable. These are the advantages of the PDD sensor:

a) The value to assemble the Custom PDD sensor costs much less compared to purchasing a whole set of HFCT. This shows that the custom sensor has a large advantage of being very affordable and easy to maintain.

b) The custom PDD sensor is really easy to be installed and used as it only needs to be positioned horizontally and very near to a PD source. Unlike HFCT which needs to be positioned at the ground braided cable of the Medium Voltage XLPE cable, the Custom PDD sensor does not have to.

c) The customized PDD Sensor is much smaller in size compared to a HFCT sensor. This is due to the design of it's Printed Circuit Board which emphasizes on functionally without having to sacrifice portability. Meanwhile, the HFCT is bulky and is quite a hassle to be installed on the Medium Voltage XLPE Cable.

d) The Custom PDD sensor is opened to a wide range of customization. Installing an artificial intelligence controlled by a microcontroller on the device is the next future step in making the sensor a SMART sensor, fulfilling the IOT aspects of futuristic design. This cannot be done on the HFCT sensor.

e) The custom PDD sensor can provide real time monitoring of PD. By using artificial intelligence, PD can be monitored in real time without having to turn off the power source of the PD. In laymen's term, the custom PDD sensor can be used for online testing of PD, while HFCT cannot. Image classification of waveforms of PD can be amended into the future AI and this can help the sensor to better detect PD intelligently.

Table 1. Maximum Average Magnitude of PD Pulses Captured by the Sensor for Each Voltage Applied

\begin{tabular}{cc}
\hline Voltage Applied $(\mathrm{kV})$ & Maximum Average Magnitude $(\mathrm{mV})$ \\
\hline 6.5 & 0.1017 \\
7.5 & 0.4024 \\
8.5 & 0.6981 \\
\hline
\end{tabular}

\section{CONCLUSION}

This paper presents an insight on the design process of the PD Detection Device prototype. The objectives of this study which are to design a functional PCB diagram and to test the functionality of the PD Detection Device have been achieved. Studies also show that by using ultrasonic sensor to detect PD, the significant magnitude values can be obtained in order to predict PD severity value with reasonable accuracy. Results of the experiment infers that the PD Detection Device is functioning as expected and that the values of PD achieved are acceptable, and the waveform obtained is a PD waveform which almost equivalent to the waveform detected by the HFCT sensor. With regards to the finished fabrication of the device, some future improvements can be done unto the device if necessary. The future improvements are installing another ultrasonic sensor on the device to improve detection range and accuracy, and embedding Artificial Intelligence into a microcontroller that can be installed onto the device, so that the device is able to carry out Image Characterization of waveforms, in order to better detect PD waveform spikes.

\section{ACKNOWLEDGEMENTS}


The authors would like to acknowledge TNB Research Sdn. Bhd. for their kind supports on the usage of the lab facilities. Special thanks also goes to those who directly or indirectly contributed to this project.

\section{REFERENCES}

[1] A. Tzimas, S. Rowland, L. A. Dissado, M. Fu \& U. H. Nilsson., "Effect of long-time electrical and thermal stresses upon the endurance capability of cable insulation material," IEEE Trans. Dielectr. Electr. Insul., vol. 16(5), pp. 1436-1443, 2009.

[2] I. Seo, U. A. Khan, J. Hwang, J. Lee \& J. Koo., "Identification of Insulation Defects Based on Chaotic Analysis of Partial Discharge in HVDC Superconducting Cable," IEEE Trans. Appl. Supercond., vol. 25(3), pp. 1-5, 2015.

[3] A. R. Mor, P. H. F. Morshuis, P. Llovera, V. Fuster \& A. Quijano., "Localization techniques of partial discharges at cable ends in off-line single-sided partial discharge cable measurements," IEEE Trans. Dielectr. Electr. Insul., vol. 23(1), pp. 428-434, 2016.

[4] N. H. Nik Ali, M. Giannakou, R. D. Nimmo, P. Rapisarda \& P. L. Lewin., "Classification and Localisation of Multiple Partial Discharge Sources Within a High Voltage Transformer Winding," in IEEE Electrical Insulation Conference (EIC), pp. 519-522, 2016.

[5] Y. O. Shaker., "Detection of partial discharge acoustic emission in power transformer," Int. J. Electr. Comput. Eng., vol. 9(6), pp. 4573-4579, 2019.

[6] F. H. Kreuger, Discharge Detection in High Voltage Equipment. 1964.

[7] Electrotechnical-sector-commitee, "IEC60270:2000, BS EN 60270:2001, High-voltage test techniques - Partial discharge measurements," Br. Stand. Board, 2000.

[8] B. Fruth and L. Niemeyer, "The importance of statistical characteristics of partial discharge data," IEEE Trans. Electr. Insul., vol. 27(1), pp. 60-69, 1992.

[9] E. Gulski, "Digital analysis of partial discharges," Dielectr. Electr. Insul. IEEE Trans., vol. 2(5), pp. 822-837, 1995.

[10] G. Ma, C. Li, X. Chen, J. Jiang, Z. Ge \& W. Chang., "Numerical sensor design for partial discharge detection on power cable joint," IEEE Trans. Dielectr. Electr. Insul., vol. 22(4), pp. 2311-2319, 2015.

[11] Z. Ahmed, G. A. Hussain, M. Lehtonen, L. Varacka \& J. Kudelcik., "Analysis of partial discharge signals in medium voltage XLPE cables," in 2016 17th International Scientific Conference on Electric Power Engineering (EPE), pp. 1-6, 2016.

[12] M. Vakilian, T. R. Blackburn, R. E. James \& B. T. Phung., "Semiconducting layer as an attractive PD detection sensor of XLPE cables," IEEE Trans. Dielectr. Electr. Insul., vol. 13(4), pp. 885-891, 2006.

[13] A. Cavallini, G. C. Montanari \& F. Puletti., "A novel method to locate PD in polymeric cable systems based on amplitude-frequency (AF) map," IEEE Trans. Dielectr. Electr. Insul., vol. 14(3), pp. 726-734, 2007.

[14] M. Tozzi, A. Cavallini, G. C. Montanari \& G. L. G. Burbui., "PD detection in extruded power cables: an approximate propagation model," IEEE Trans. Dielectr. Electr. Insul., vol. 15(3), pp. 832-840, 2008.

[15] F. P. Mohamed, W. H. Siew, J. J. Soraghan, S. M. Strachan \& J. Mcwilliam., "The use of power frequency current transformers as partial discharge sensors for underground cables," IEEE Trans. Dielectr. Electr. Insul., vol. 20(3), pp. 814-824, 2013.

[16] A. Rodrigo, P. Llovera, V. Fuster \& A. Quijano., "High performance broadband capacitive coupler for partial discharge cable tests," IEEE Trans. Dielectr. Electr. Insul., vol. 20(2), pp. 479-487, 2013.

[17] C. D. Johnson, "Process Control Instrumentation Technology". 2014.

[18] M. S. K. M. H. M. Sharif, N. A. M. Jamail \& N. A. Othman., "Analysis of Electric Field and Current Density on XLPE Insulator," Int. J. Electr. Comput. Eng., vol. 7(6), pp. 3095-3104, 2017.

[19] D. Evagorou, A. Kyprianou, P. L. Lewin, A. Stavrou, V. Efthymiou \& G. E. Georghiou., "Evaluation of partial discharge denoising using the wavelet packets transform as a preprocessing step for classification," Annu. Rep.Conf. Electr. Insul. Dielectr. Phenom., pp. 387-390, 2008.

[20] D. Evagorou, A. Kyprianou, G. E. Georghiou, L. Hao, P. L. Lewin \& A. Stavrou., "Multisource PD identification based on phase synchronous and asynchronous data," Annu. Rep. Conf. Electr. Insul. Dielectr. Phenom., pp. 460-463, Oct 2011.

[21] A. Contin, A. Cavallini, G. C. Montanari, G. Pasini \& F. Puletti., "Digital detection and fuzzy classification of partial discharge signals," IEEE Trans. Dielectr. Electr. Insul., vol. 9(3), pp. 335-348, Jun 2002.

[22] N. H. Nik Ali, J. A. Hunter, P. Rapisarda \& P. L. Lewin., "Identification of Multiple Partial Discharge Sources in High Voltage Transformer Windings," IEEE Conf. Electr. Insul. Dielectr. Phenom., pp. 188-191, 2014.

[23] N. H. Nik Ali, M. S. Abd Rahman, J. A. Hunter, P. Rapisarda \& P. L. Lewin., "Wavelet and Mathematical Morphology as the de-noising methods for PD analysis of high voltage transformer windings," IEEE Electr. Insul. Conf., pp. 214-217, 2015.

[24] X. Ma, C. Zhou \& I. J. Kemp., "Interpretation of wavelet analysis and its application in partial discharge detection," IEEE Trans. Dielectr. Electr. Insul., vol. 9(3), pp. 446-457, 2002.

[25] S. Sriram, S. Nitin, K. M. M. Prabhu \& M. J. Bastiaans., "Signal denoising techniques for partial discharge measurements," IEEE Trans. Dielectr. Electr. Insul., vol. 12(6), pp. 1182-1191, 2005.

[26] X. Zhou, C. Zhou \& I. J. Kemp., "An improved methodology for application of wavelet transform to partial discharge measurement denoising," IEEE Trans. Dielectr. Electr. Insul., vol. 12(3), pp. 586-594, 2005.

[27] A. Z. Abdullah et al., "Wavelet based de-noising for on-site partial discharge measurement signal," Indones. J. Electr. Eng. Comput. Sci., vol. 16(1), pp. 259-266, 2019. 


\section{BIOGRAPHIES OF AUTHORS}
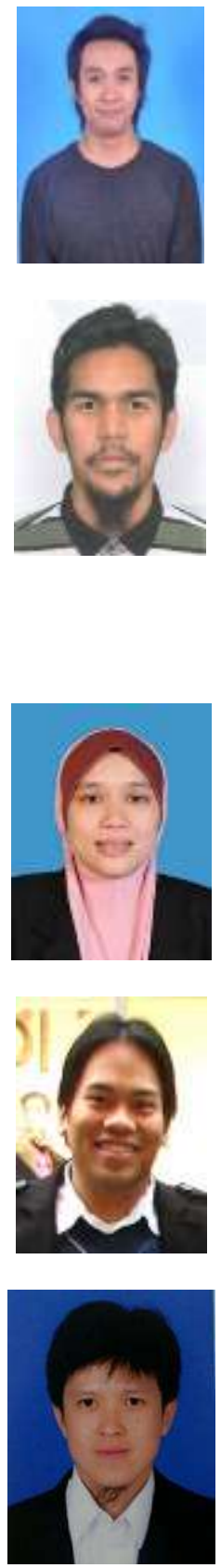

Mohamad Izmir Farhan Mohamad Radzi is currently a final year and final semester student majoring in B.Eng Electrical Power, in Universiti Tenaga Nasional (UNITEN). He started his degree in Virginia Polytechnic Institute and State University in the United States of America back in 2014. He completed his internship in TNB Research Sdn. Bhd recently and is working on a Partial Discharge project under his supervisor (Assoc. Prof. Dr. Azrul bin Mohd Ariffin).

Nik Hakimi Nik Ali was born in Malaysia in 1990. He received the B.Eng. degrees in Electrical Power Engineering (First Class Honors) from the Universiti Tenaga Nasional (UNITEN), Malaysia in 2013. He received the Ph.D. degrees in Electronics and Electrical Engineering from the University of Southampton, UK in 2017. He worked as a Research Fellow in School of Electronics and Computer Science in University of Southampton, UK from 2018 to 2019. Currently, he is a Post-doctoral Researcher in Institute of Power Engineering (IPE) in UNITEN, Malaysia. He is also a graduate member of Board of Engineers Malaysia (BEM). His research interests are within the generic areas of applied signal processing. Within high voltage engineering this includes condition monitoring of high voltage cables and transformers, partial discharge measurement, HV insulation/dielectric materials, transformer rating analysis and applied signal processing.

Norhidayu Rameli was graduated from Universiti Putra Malaysia with B.Eng degree in Electrical and Electronic in 2010. In 2013 and 2017, she completed her studies in MSc and PhD, respectively in Electrical Power Engineering. During MSc and PhD programmed, she had been tutoring courses including Power System labaratory, Engineering Mathematics, Electrical Circuit and involved with local and international conferences. Currently she is working in TNB Research Sdn Bhd as a researcher.

Azrul Mohd Ariffin was born in Malaysia in 1981. He received his B.Eng in Electrical Engineering (First Class Honors) from University of Southampton, UK in 2004. He then pursued his doctorate at the same university and was awarded the Ph.D degree in 2008. His thesis investigated the electroluminescence phenomenon in insulating polymers subjected high electric field. Currently, he is an Associate Professor and the Director of Programme Management Office at Universiti Tenaga Nasional (UNITEN), Malaysia. He is also a Professional Engineer (P.Eng) registered with Board of Engineers, Malaysia (BEM).

Muhamad Safwan Abd Rahman received his Master of Engineering in Electrical Power and Ph.D in Electrical Engineering from Universiti of Southampton, United Kingdom in 2010 and 2014 respectively. He is currently a Senior Lecturer in Department of Electrical and Electronics Engineering, Universiti Tenaga Nasional (UNITEN), Malaysia. His research interests are High Voltage Engineering, Power Transformers \& Cables, Partial Discharge studies, Lightning Protection studies, Thermal Stress studies, Statistical Pattern Recognition, Finite Element Analysis using CFD \& Electrostatic Method and Insulation Coordination. He had conducted various research and consultancy works since joining UNITEN in 2014 and he is currently supervising two Ph.D candidates, 1 Master candidate and undergraduate students who are working in various fields in High Voltage. He is also actively involved in Occupational Safety and Health $(\mathrm{OSH})$ activities and a member of OSH in Faculty of Engineering, UNITEN which handling safety and policies. He is also a member of IEEE and Dielectrics Electrical and Insulation Society (DEIS), a member of Institution of Engineering and Technology (IET), a member of Malaysian High Voltage Network (MyHVnet) and a member of Institute of Power Engineering (IPE), UNITEN. 



Dr. Mohamad Radzi Ahmad received his BSc. in Electrical Engineering from Purdue University School of Engineering and Technology Indianapolis USA in 1991, MSc. in Electronics System Design Engineering from Universiti Sains Malaysia in 2010, and $\mathrm{PhD}$ in Electrical and Electronics Engineering from Universiti Teknologi PETRONAS Malaysia in 2014. He joined Universiti Teknologi PETRONAS in 2016, where he is currently a Senior Lecturer in the Electrical and Electronic Engineering Department. Prior to joining UTP, Dr Radzi had 20 years of professional experience with electronic industries such as Intel, Avago, Western Digital and Fairchild Semiconductor, and held various positions ranging from Senior Manager to Product R\&D Engineer.

Ali Syari'ati Mohd Salleh received his BSc. in Electrical Engineering from Universiti Teknologi Mara in 2009, Msc. In Computer and Microelectronics system from Universiti Teknologi Malaysia in 2015. He joined Intel Microelectronic system as Analog SoC Validation, SoC HW Validation, IOT, Machine Learning and Deep Learning Engineer from 2009 until 2019. 